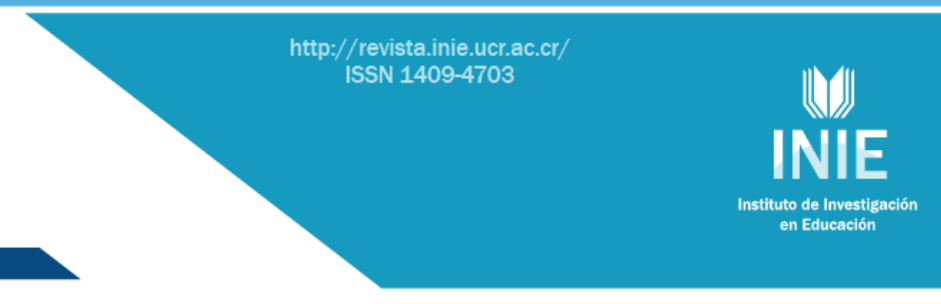

\title{
LA VINCULACIÓN INVESTIGACIÓN-DOCENCIA-SOCIEDAD: EL CASO DE PAUL EHRENFEST
}

LINKING RESEARCH-TEACHING-SOCIETY: THE CASE OF PAUL EHRENFEST

\author{
Volumen 12, Número 3 \\ Setiembre-Diciembre
}

pp. 1-22

Este número se publicó el 30 de setiembre de 2012

Ricardo Guzmán Díaz

José Antonio Cervera Jiménez

Revista indizada en REDALYC

Revista distribuida en las bases de datos:

CATÁLOGO DE LATINDEX, IRESIE, CLASE, DIALNET, DOAJ, E-REVIST@S,

Revista registrada en los directorios:

ULRICH'S, REDIE, RINACE, OEI, MAESTROTECA, PREAL, CLASCO 


\title{
LA VINCULACIÓN INVESTIGACIÓN-DOCENCIA-SOCIEDAD: EL CASO DE PAUL EHRENFEST \\ LINKING RESEARCH-TEACHING-SOCIETY: THE CASE OF PAUL EHRENFEST
}

\author{
Ricardo Guzmán Díaz ${ }^{1}$ \\ José Antonio Cervera Jiménez
}

\begin{abstract}
Resumen: El presente ensayo trata sobre el pensador Paul Ehrenfest, su labor científica, académica, social y humanística. Fue un científico comprometido y crítico con los cambios vertiginosos que vivió la física a principios del siglo pasado, y un profesor destacado, que ayudó a crear una escuela de física importante en Holanda cuya experiencia académica contribuyó a la formación de científicos que destacaron en sus disciplinas en las siguientes décadas. En sus últimos años fue precursor de la vinculación entre la universidad, la industria y las necesidades sociales.
\end{abstract}

Palabras clave: PAUL EHRENFEST, BIOGRAFÍA, FÍSICA, MECÁNICA CUÁNTICA, SOCIEDAD

\begin{abstract}
This essay deals about Paul Ehrenfest and his scientific, academic, social and humanistic labor. He was a committed scientist and very critic of the rapid changes that physics experienced in the early last century, and an outstanding professor, who helped create an important Physics school in the Netherlands whose academic experience contributed to the training of scientists who excelled in their fields in the following decades. In his later years he pioneered the linkage between university, industry and social needs.
\end{abstract}

Keywords: PAUL EHRENFEST, BIOGRAPHY, PHYSICS, QUANTUM MECHANICS, SOCIETY.

\footnotetext{
1 Doctor en Estudios Humanísticos con especialidad en Ciencia y Cultura. Docente e investigador del Tecnológico de Monterrey, México. Miembro de la cátedra de investigación en Ciencia y Cultura del Tecnológico de Monterrey. Dirección electrónica: rguzman@itesm.mx

2 Doctor en Ciencias Físicas por la Universidad de Zaragoza y doctor en Estudios de Asia y África, especialidad China, por el Colegio de México. Profesor-investigador del Colegio de México, México. Dirección electrónica. jacervera@colmex.mx
}

Ensayo recibido: 13 de febrero, 2012

Aprobado: 6 de agosto, 2012 


\section{Introducción}

A principios del siglo $\mathrm{XX}$, la nueva física fue surgiendo a partir de las tensiones entre diversos paradigmas clásicos de explicación. Particularmente los trabajos de Boltzmann, de Planck y de Einstein son muy representativos de los esfuerzos por reconciliar las visiones mecánica, termodinámica y electromagnética del mundo físico. Sin embargo, hasta 1911, año en que se organiza la primera conferencia Solvay, en la cual se reconoce la urgencia de poner en claro el papel que los nuevos cuantos de acción deberían jugar en la física, eran pocos los científicos que estaban al tanto de las amenazas que el trabajo de Planck y de Einstein representaba a los puntos de vista tradicionales en la física y todavía menos los que se habían preocupado por clarificar los alcances de los nuevos conceptos en torno al cuanto. Paul Ehrenfest (1880-1933), físico austriaco que se encontraba viviendo en Rusia y quien pronto sustituiría al afamado Lorentz como profesor de física teórica en Leiden, fue uno de los pocos interesados en tomar con seriedad dichos trabajos, especialmente los artículos de Planck, y tratar de definir su relación con la vieja física. A lo largo de varios años, Ehrenfest fue dejando su marca en el desarrollo de la teoría cuántica en sus varias fases.

Ehrenfest fue amigo cercano de Einstein e influyó de muy diversas maneras en su disciplina, a la que se dedicó con verdadera pasión. Martin Klein nos hace un retrato fugaz de la contribución de Ehrenfest a la física basándose en una cita de Maxwell que intenta mostrar a la ciencia como algo vivo en la que no solo un descubrimiento, sino también la identificación de una paradoja o el uso de una frase científica o la exposición integradora de una teoría son parte de la dinámica y la construcción de la misma (Klein, 1981, p. 3). Ehrenfest descubrió lo que más tarde Einstein llamó el principio adiabático, jugó con infinidad de paradojas (Klein, 1959, pp. 128, 136, 146, 154, 161, 303, 410, 479, 539), ${ }^{3}$ acuñó el término 'catástrofe del ultravioleta', que con tanto tino exponía una de las limitaciones explicativas de la física clásica, y expuso, con maestría, junto con su esposa Tatiana, el estado del arte en que se encontraba la mecánica estadística en la primera década del siglo $X X$, en un tratado que se convirtió en un clásico y en una referencia obligada para los estudiosos de esa disciplina.

${ }^{3}$ De los trabajos científicos de Ehrenfest, varios de ellos están centrados en la presentación, formulación o identificación de alguna paradoja resultante dentro de los esquemas teóricos aceptados hasta ese momento dentro de la disciplina de la física. 
Ehrenfest se convirtió en punto de enlace importante en la red de científicos. Fue muy apreciado por su talento para la discusión y el debate. Aprovechaba cualquier oportunidad para señalar los puntos débiles, las falacias y las paradojas de un concepto o teoría, y sus publicaciones fueron siempre dirigidas hacia los fundamentos de la física. Su capacidad crítica fue reconocida por la comunidad científica y su trabajo tuvo gran impacto en el surgimiento de la teoría cuántica. Además, sus discípulos recibían de él mucho más que lecciones de física, pues él ofrecía (aunque también requería de ellos), una dedicación total. Nos dice Martin Klein que "aunque sea fácil pensar que la física teórica tiene que ver exclusivamente con conceptos abstractos lejanos a los asuntos humanos ordinarios, Ehrenfest enseñaba y vivía la física con pasión" (Klein, 1989, p. 30). En este artículo ofrecemos una breve semblanza de Paul Ehrenfest en su vida personal, en su pasión por la física y en su sentido de compromiso como guía de futuros científicos, aspectos que para él eran casi literalmente una misma cosa. Discutiremos también el papel que le tocó jugar a Ehrenfest en la conformación de una visión respecto a la responsabilidad social de la universidad.

Para este propósito tomamos en consideración a diferentes estudiosos de la historia de la ciencia que se han preocupado por rescatar el legado de Paul Ehrenfest. Probablemente el más importante es Martin Klein, quien a través de una recolección de los trabajos de Ehrenfest (1959), un libro biográfico (1985) y varios artículos (1981, 1989) nos retrata diversos aspectos de la vida personal y profesional de Paul Ehrenfest. Otros historiadores han abordado aspectos más específicos, en relación con su lucha científica en un periodo particular de la vida de Ehrenfest (Boeyink, 2005), sobre la historia de su cátedra en Leiden (Lunteren, 2003) o sobre la contribución de Ehrenfest en el desarrollo institucional de la física en Holanda (Lunteren, 2004). Consideramos que el presente trabajo constituye una contribución adicional que aporta una visión integradora de la función de Paul Ehrenfest como investigador, docente y promotor de una visión social de la ciencia que, a la distancia, lo presenta como precursor remoto de los modelos de vinculación academia-industriaestado-sociedad.

\section{Años de formación}

Paul Ehrenfest nació el 18 de enero de 1880 en Viena y creció en la misma ciudad en el seno de una familia judía originaria de un pueblo localizado en Moravia. Fue el menor de 5 
hermanos, de los cuales el mayor, Arthur, quien estudió ingeniería mecánica, tuvo gran influencia en despertar el interés del pequeño Paul por los rompecabezas matemáticos y por entender el funcionamiento de diversos dispositivos; fue gracias a su hermano mayor que Paul se familiarizó, desde niño, con ciertos conceptos físicos y científicos. En su familia no se seguían las tradiciones judías, pero no por eso dejaron de ser víctimas de discriminación y abuso. Paul desde pequeño conoció de la existencia de otras religiones, sus confrontaciones, las hipocresías en torno a ellas y desde los doce años abandonó la fe y le daba gran placer discutir y argumentar sobre lo absurdo y la falsedad de todas las religiones organizadas (Klein, 1985, pp. 27-29).

Los tiempos de sus primeros estudios fueron particularmente difíciles para el pequeño Paul, sobre todo por la pérdida de sus padres. Su madre murió en 1890 y su padre seis años más tarde. Ehrenfest sufrió en su niñez de severas depresiones relacionadas con amargas experiencias en la escuela, por lo que tiempo después, como padre, insistiría en educar a sus propios hijos en casa (Klein, 1985, p. 34).

Ehrenfest continuó en 1899 sus estudios en la Escuela Superior Técnica de Viena (Technische Hochschule), aunque también tomó cursos en la Universidad. Aunque se inscribió en química como su campo principal, muy pronto se vio atraído por la física teórica guiado por Ludwig Boltzmann, quien le transmitió la esencia y el espíritu de esa disciplina (Klein, 1985, p. 38). Era costumbre que los estudiantes en países de habla germana visitaran durante sus estudios varias universidades y Ehrenfest también siguió ese patrón. En 1901 se trasladó a Gotinga, donde permanecería año y medio.

Su estancia en Gotinga fue muy enriquecedora. Para Ehrenfest, Gotinga resultó un lugar de estudio ideal por sus actividades de enseñanza, la camaradería, la presencia de muchos compañeros de otros países y sobre todo por las intensas discusiones que ocurrían durante las reuniones semanales de los estudiantes. Fue ahí donde Ehrenfest desarrolló su independencia científica y se preparó para el inicio de sus propias investigaciones. Entre los compañeros extranjeros se encontraba una joven matemática, Tatiana Alexeyevna Afanassjewa, originaria de San Petersburgo. Era algunos años mayor que Ehrenfest, pero tenía, al igual que él, un fuerte espíritu independiente y, sobre todo, una fascinación por los fundamentos de las ciencias exactas. Además, ambos sentían la misma aversión por el alcohol, el tabaco u otros estimulantes. Después de dos meses de haber llegado a Gotinga 
decidieron casarse, aunque esperarían primero a terminar sus estudios (Lunteren, 2003, p.2).

Habiendo terminado su estancia en Gotinga, Ehrenfest realizó una corta visita a Leiden en la primavera de 1903, donde, junto con Walter Ritz, de quien se había hecho amigo, tomó unos cursos con Lorentz. Difícilmente podría haber imaginado Ehrenfest que Leiden se convertiría en el centro de su vida en menos de una década. Al término de esos cursos regresó a Viena para terminar sus estudios bajo la dirección de Boltzmann. Terminó su doctorado en junio de 1904 y en seguida se casó con Tatiana allí mismo, en Viena, pero con el plan de vivir, en un futuro, en la tierra natal de su esposa. Las leyes Austro-Húngaras prohibían el matrimonio entre judíos y cristianos, por lo cual ambos tuvieron que declararse como no practicantes de religión alguna. En el verano de ese año visitaron Rusia con la intención de orientarse y planear su vida futura en ese país, pero Viena fue su lugar de residencia por casi dos años. En octubre de 1905 tuvieron a su primera hija, a quien llamaron igual que su madre (Klein, 1985, pp. 45-52).

Después de doctorarse, Ehrenfest continuó estudiando en la Universidad de Viena, asistiendo al seminario de Boltzmann y empezando a desarrollar sus propias ideas en torno a la mecánica estadística y su conexión con la teoría de la radiación de Planck. En ese momento no era prioritario encontrar una posición académica con ingreso fijo, pues él y Tatiana podían vivir de pequeños ingresos que habían heredado, aunque Ehrenfest tenía la esperanza de obtener una posición académica en Gotinga. Con esa idea en mente, en la primavera de 1906 dejaron Viena, pasaron el verano en Suiza y posteriormente llegaron a Gotinga, donde fueron recibidos con la noticia del suicidio de Boltzmann, lo que debió ser un golpe muy duro para Ehrenfest. Su esperanza de obtener un puesto parecía ciertamente lejana, pero el matemático Félix Klein lo invitó a participar en un seminario. Eligió hablar sobre el carácter estadístico de la segunda ley de la termodinámica. Su presentación tuvo gran éxito, lo que convenció a Félix Klein de invitar a Ehrenfest a contribuir, si lo deseaba con la participación conjunta de su esposa, con un artículo sobre la mecánica estadística que formaría parte de la Enciclopedia Matemática que Klein estaba organizando. Este proyecto le tomaría casi tres años poder concluirlo.

En el otoño de 1907, Paul y Tatiana decidieron que ya era momento de llevar a cabo sus planes de mudarse a San Petersburgo. Ehrenfest nunca sintió a Viena como su hogar y el lugar de origen de su esposa era la alternativa como lugar de residencia estable. Ehrenfest 
buscó tener contacto directo con los físicos del lugar, y uno de ellos, Abram Fedorovitch Joffe, se convirtió en amigo de toda la vida. ${ }^{4}$

El clima intelectual en la Universidad de San Petersburgo era muy pobre y difería enormemente del que se podía encontrar en Gotinga. La investigación original no era estimulada. Ehrenfest trató de dotar de un nuevo espíritu a la física en San Petersburgo organizando conferencias informales cada dos semanas, por lo general en su propia casa, lo que fue un antecedente del coloquio que años después establecería en Leiden. Sin embargo, cinco años después, cuando la situación académica en Rusia, bajo los ordenamientos del nuevo ministro de educación, se deterioró aún más, Ehrenfest tomó la decisión de abandonar Rusia para buscar nuevos horizontes (para mayores detalles sobre la estancia de Ehrenfest en Rusia, véase Klein, 1985, pp. 83-90).

En enero de 1912, Ehrenfest empezó un recorrido por las principales universidades de habla alemana. Ya para entonces contaba con varias publicaciones importantes. Dentro de la comunidad de físicos teóricos su nombre era suficientemente conocido y su trabajo contaba con buena reputación. Sin embargo, no tuvo éxito en encontrar una posición como profesor. En Leipzig tenía a un buen amigo, el matemático Gustav Herglotz, y aunque la universidad de esa ciudad no tenía mucho atractivo para Ehrenfest, la sola presencia de Herglotz era suficiente para él, pero desgraciadamente no contaba con las credenciales exigidas, que consistían en haber obtenido un doctorado de una universidad alemana. Otra posibilidad era Munich, donde Sommerfeld, quien había sido asistente de Félix Klein, había adquirido una gran reputación. Sin embargo tampoco hubo suerte en ese lugar. Las posibilidades en Praga eran mejores. Allí conoció a Einstein, con quien solo había tenido alguna breve comunicación previa, pero ese encuentro sería el inicio de una larga y poderosa amistad. Tenían casi la misma edad, compartían un mismo contexto judío, tenían los mismos intereses científicos y además compartían su aversión a las formalidades (Lunteren, 2003, p. 5). Einstein estaba a punto de dejar su puesto en Praga para regresar a Zurich y consideró que Ehrenfest era la persona ideal para sucederlo en la vacante que dejaría. Sin embargo, la rectitud de Ehrenfest canceló esta opción. Su negativa a abandonar su declaración previa, cuando se casó con Tatiana, en el sentido de no adherirse a ninguna

\footnotetext{
4 Joffe nació, igual que Ehrenfest, en 1880 , y también provenía de una familia judía. Colaboró en Munich con W. K. Roentgen y se doctoró en 1906. En 1912 formó el seminario de física de San Petersburgo junto con Piotr Leonidovich Kapitza, Jacob llich Frenkel y el mismo Paul Ehrenfest.
} 
religión, lo eliminaba como candidato según las rígidas regulaciones de la Universidad (Klein, 1985, p. 178). Afortunadamente, a su regreso a San Petersburgo lo aguardaba una agradable sorpresa, una invitación de Lorentz a sucederlo como profesor de física teórica en Leiden, lugar donde residiría el resto de sus días. Lorentz había considerado en primera instancia para su sucesión a Einstein y al joven y brillante Peter Debye, pero ante la imposibilidad de que ellos lo sucedieran, y apreciando la claridad del trabajo de Ehrenfest y el hecho de que sus intereses fueran muy cercanos a los suyos, en temas como la teoría de la relatividad, la mecánica estadística y la reciente problemática cuántica, no dudó en invitarlo. El 25 de septiembre de 1912 fue nombrado catedrático de física teórica (Jungnickel, et al., 1990, p. 254) en la Universidad de Leiden, donde ejercería un fructífero profesorado por más de veinte años (para mayores detalles sobre la sucesión de Lorentz, véase Lunteren 2003, pp. 6-9).

\section{Ehrenfest como profesor y como crítico de su disciplina}

Paul Ehrenfest se distinguió desde el principio por sus cualidades pedagógicas y críticas. Los estudiantes que podían seguirle el paso se beneficiaban en gran medida del trato personal que les ofrecía su maestro. Ya en su conferencia inaugural de diciembre de 1912 en Leiden, hacía él mismo referencia al hecho de que durante su presencia allí, buscaría el contacto personal con sus estudiantes

¡Damas y caballeros estudiantes! Mis deberes para con ustedes los entiendo de la siguiente manera: Deberé contribuir con todos mis conocimientos y habilidades a que ustedes encuentren, con el menor daño posible, el camino que corresponda a la esencia de sus talentos. Las lecciones sistemáticas que sostendré para ustedes sobre las diferentes disciplinas y problemas particulares de la física teórica deben ser un medio necesario pero de ninguna manera suficiente para aproximarse a esa meta. Sobre todo me será necesario entrar en contacto personal con cada uno de ustedes. Les pido ver en mí un camarada mayor y no un hombre que se encuentra en una etapa diferente de desarrollo científico. (Klein, 1959, p. 327)

\footnotetext{
${ }^{5}$ La disciplina llamada 'física teórica' como tal era relativamente nueva. Su equivalente anterior era la física matemática, pero con el nuevo nombre se hizo más énfasis en la intuición física que en la abstracción matemática, dándole a esa disciplina un status más autónomo. Este proceso de cambio disciplinar fue reforzado por el desarrollo de la teoría de la relatividad y los posteriores desarrollos dentro de la mecánica cuántica.
} 
Lorentz reconocía, poco después de esa conferencia, que Ehrenfest había logrado en poco tiempo lo que él había intentado, pero nunca conseguido, en su profesorado: hacer hablar a los estudiantes. Estos últimos podían ahora expresarse con toda confianza y Ehrenfest compartía con ellos sus mismas pasiones y sus mismas áreas de interés. La física y la vida fuera de la física no eran cosas diferentes para Ehrenfest. Las preguntas que cada estudiante se planteaba sobre su existencia, Ehrenfest las asumía también (Lunteren, 2003, pp. 12-13). ${ }^{6}$ El estilo de enseñanza de Lorentz se caracterizaba por sus lecciones formales y perfectamente preparadas. En cambio, Ehrenfest enseñaba de manera mucho más informal e intensa, involucrando a los alumnos en la física del momento, discutiendo asuntos concernientes a las más recientes publicaciones (Boeyink, 2005, p. 61). Les daba mucha mayor importancia a los conceptos cruciales que a la continuidad de los argumentos y las discusiones y las preguntas eran para él un componente esencial de la ciencia (Boeyink, 2005, p. 62).

Sin embargo, la buena impresión que los alumnos de Ehrenfest tenían sobre él, no impedía que él mismo dudara siempre de sus cualidades. La imagen negativa que tenía de sí mismo se evidencia en muchas de sus comunicaciones con sus colegas. Ehrenfest argumentaba en una de esas cartas, dirigida tanto a Lorentz como a Debye, que este último habría sido mucho mejor elección como sucesor de Lorentz. En otra carta dirigida a su alumno Jan Burgers se refería a la brecha generacional que sentía de manera cada vez más intensa: "Siento ahora que pierdo rápidamente todo contacto con los más jóvenes y me siento más viejo - cuál es la causa y cuál el efecto es algo que no sé" (citado en Boeyink, 2005, p. 61).

Desde el principio, a su llegada a Leiden, puso toda su atención y energía en la educación de sus alumnos, tratando de crear un ambiente intelectual como el que le había tocado vivir en sus tiempos de estudiante en Gotinga. El problema principal que advirtió fue la falta de contacto entre estudiantes y profesores por diferentes razones profesionales, sociales y de diferencias de edad. Una de sus primeras iniciativas estuvo dirigida a promover una comunicación más abierta. Estableció un coloquio semanal que se convertiría en algo

\footnotetext{
${ }^{6}$ Esta relación tan personal con sus alumnos también tenía, por supuesto, su lado negativo. Ehrenfest interfería seriamente con la vida de sus alumnos y en ocasiones incluso los hacía cargar con sus propias depresiones y dudas existenciales.
} 
esencial en el calendario de los físicos holandeses ${ }^{7}$ y que con el tiempo tuvo una gran influencia en el estilo de hacer física teórica en todo el mundo. En estas reuniones semanales, Ehrenfest logró reunir estudiantes, profesores e investigadores para escuchar ponencias sobre nuevas investigaciones y, sobre todo, para discutirlas. La presencia activa de Ehrenfest fue lo que distinguió estas reuniones de sus contrapartes en otras universidades, encargándose siempre de hacer preguntas que llevaran la discusión hacia los puntos esenciales del tema tratado y planteando sugerencias o reformulaciones de lo presentado. Uno de sus alumnos, Uhlenbeck, quien se convertiría en un destacado físico, resumió la esencia de las reuniones y del papel de Ehrenfest en las mismas de la siguiente manera: "Era una experiencia educativa (a veces un poco dolorosa si tú eras el orador) escuchar a Ehrenfest resumir la discusión y en ocasiones toda la plática, de tal manera que al final, todos, el orador incluido, entendían de qué se había tratado" (Uhlenbeck, 1956, p.432). Los estudiantes podían asistir por invitación expresa de Ehrenfest, quien requería de ellos asistir regularmente, pues de lo contrario ya no eran admitidos, pues para Ehrenfest, el no asistir con regularidad una vez que un alumno era invitado, era un claro indicador de que el alumno carecía de un compromiso total hacia la física, tal como Ehrenfest lo demandaba. Además del coloquio, Ehrenfest también estableció un cuarto de lecturas exclusivo para las ciencias físicas que, en su visión, debería contener los textos y tratados principales, la colección de obras de los físicos más destacados y las revistas más importantes (Klein, 1985, pp. 9-10 y Klein, 1989, pp. 35-37).

Su especial talento para dar clases se había manifestado desde antes de llegar a Leiden. Durante su estancia en Rusia, aunque nunca tuvo un puesto regular como profesor, sí enseñó oficialmente un curso en 1910 en el Instituto Politécnico de San Petersburgo sobre ecuaciones diferenciales en la física matemática. Para su amigo Joffe aquellas lecturas fueron maravillosas, y las describiría muchos años después comentando que "ninguna presentación de esta disciplina clásica había combinado jamás la física y las matemáticas en semejante unidad armoniosa" (Klein, 1985, p. 89). Algún tiempo después, ya en funciones como profesor en Leiden, enseñaba alternadamente dos cursos, uno sobre teoría electromagnética que finalizaba con la teoría del electrón de Lorentz y la teoría de la relatividad de Einstein, y otro sobre mecánica estadística que concluía con las nuevas ideas

${ }^{7}$ Dicho coloquio sigue existiendo en la actualidad, se le denomina Colloquium Ehrenfestii y sigue teniendo lugar, al igual que cuando lo inició Ehrenfest, los miércoles por la noche. 
en torno a la teoría cuántica, impregnando así a sus cursos de la física del momento, de la física que se estaba haciendo y que era motivo de vigorosos debates que Ehrenfest promovía en su proceso de enseñanza. Las lecciones de Ehrenfest brillaban sobre todo por su viveza condimentada por metáforas y por su dominio incompleto del holandés (Ehrenfest, 1959, p. XI).

De acuerdo con Uhlenbeck, lo que hacía que las lecciones de Ehrenfest fueran tan excelentes, era en parte su claridad. Siempre se esforzaba por reconocer y resaltar el punto crucial del argumento. El énfasis siempre aparecía en las ideas físicas y la estructura lógica de la teoría. Tal vez sus alumnos no aprendían mucho "cómo calcular", pero sí aprendían "cuál era la esencia del problema", pues "solo los fundamentos se desarrollaban cuidadosamente y se taladraban en nuestra mente" (Uhlenbeck, 1956, p. 432).

No era fácil llegar a ser estudiante doctoral de Ehrenfest, y de hecho procuraba no motivarlos mucho cuando no estaba convencido de sus cualidades. Para ser aceptado no era suficiente tener talento, sino también una entera vocación por la disciplina. De hecho, Ehrenfest promovió solo una decena de estudiantes, la mayoría de los cuales destacaron en gran medida en el mundo de la ciencia. La historia de algunos de ellos nos permitirá conocer también un poco más sobre el maestro.

En el periodo de 1918 a 1922, Ehrenfest promovió al grado doctoral a varios estudiantes (entre ellos los más destacados fueron Burgers, Kramers y Coster). El primero de ellos, Jan Burgers (cf. The Burgers Program for Fluid Dynamics), ${ }^{8}$ llegó a Leiden en el otoño de 1914. Ehrenfest reconoció de inmediato su capacidad matemática. En un principio se interesó por la física experimental trabajando en el laboratorio de Kamerlingh Onnes, pero después se orientó más por la teoría, donde dio continuidad a una de las principales aportaciones de Ehrenfest en torno a su principio adiabático. Burgers reconocía el talento de Ehrenfest como guía en el desarrollo científico de sus estudiantes

Ehrenfest nos enseñaba cómo leer artículos científicos, identificar los supuestos hechos por el autor, y descubrirlos cuando no eran dados en forma explícita. Su

\footnotetext{
${ }^{8}$ Jan Burgers escribió su disertación bajo la dirección de Ehrenfest en 1918 sobre el modelo atómico de Rutherford-Bohr, completando el trabajo de Ehrenfest sobre la conexión entre las reglas de cuantización de Bohr-Sommerfeld y las variantes adiabáticas. Fue profesor en Delft, donde se convirtió en un experto en dinámica de fluidos. Más tarde formó parte de la facultad de la Universidad de Maryland, donde desarrolló su interés sobre la relación de la ecuación de Boltzmann con las ecuaciones de la dinámica de fluidos. Además de su trabajo meramente científico, se interesó también por temas de interés social y/o filosófico más amplios.
} 
poderosa mente analítica abría nuestros ojos a muchas sutilezas de la teoría física. Siempre se esforzó por encontrar interpretaciones de nuevas ideas y tenía medios efectivos para la ilustración de sus peculiaridades (...) Ehrenfest abarcaba y enseñaba física teórica como un todo. (citado en Klein, 1985, p. 209)

Contemporáneo de Burgers, Hendrik Anthony Kramers, aunque en un principio no calificaba como físico, según la apreciación de Ehrenfest, por sus amplios intereses que le impedían una dedicación exclusiva a la física, al final se convirtió en su sucesor en Leiden en 1934. Un discípulo más de Ehrenfest que con el tiempo adquirió gran renombre fue Dirk Coster. Ehrenfest lo encaminó en el estudio de la espectroscopía y dirigió su tesis sobre Espectros de rayos $X$ y la teoría atómica de Bohr. Más tarde, trabajando junto con Bohr en Copenhague, sentó las bases para el futuro descubrimiento del elemento hafnio. Posteriormente se desempeñó como profesor de física y metrología en la Universidad de Gröningen.

Poco después del periodo de guerra, Ehrenfest promovió al grado doctoral a varios estudiantes más (entre ellos se cuentan Uhlenbeck, Goudsmit, Tinbergen y Casimir). Uhlenbeck y Goudsmit fueron invitados por Ehrenfest en 1921 al coloquio de los miércoles. A Goudsmit le interesó el tema de la estructura fina del espectro. Siguiendo el consejo de su maestro, pasó dos meses con Paschen en Tubinga para aprender la espectroscopia experimental. En 1924 trabajó también con Zeeman en su laboratorio de Ámsterdam, donde pudo especializarse un poco más en esa área. Presentó su examen doctoral en 1925 y Ehrenfest lo mandó unas semanas con Bohr a Copenhague. Uhlenbeck era un poco mayor. Por un tiempo fue a tomar algunas clases a Roma, donde conoció a Enrico Fermi. Al regresar a Holanda siguió la recomendación de Ehrenfest de que profundizara, junto con Goudsmit, en el tema de las líneas espectrales, cooperación que fue muy exitosa, dando por resultado la hipótesis del spin del electrón, paso de gran importancia en el desarrollo de la teoría de los espectros atómicos (Goudsmit, 1971). ${ }^{9}$ Uhlenbeck, según parece, heredó algunas de las virtudes de su maestro a juzgar por la siguiente cita de Cohen, alumno de Uhlenbeck, al referirse a él

\footnotetext{
${ }^{9}$ Existe una narración del descubrimiento del spin del electrón dada por el mismo Goudsmit y que después fue transcrita y publicada.
} 
[Uhlenbeck] me advertía con frecuencia que en lugar de tratar de ser original, era mucho más importante ser claro y correcto y resumir críticamente el estado presente de un campo, en la misma tradición que Ehrenfest. Sabiamente observaba que lo que con frecuencia tiene valor duradero no es la primera contribución original a un problema, sino más bien la presentación final escrita clara y críticamente. (Cohen, 1990, p. 621)

Esto nos habla de la fuerte influencia de Ehrenfest en sus discípulos y la trascendencia de sus enseñanzas.

Un alumno muy especial de Ehrenfest fue quien años después se convertiría en el primer premio Nobel de economía. Nos referimos a Jan Tinbergen, quien estudió primero física teórica bajo la dirección de Ehrenfest. Tinbergen también apreciaba mucho a sus maestros

A Ehrenfest le debo mucho. Estudié física en una época en la que se encontraban juntas varias personas fascinantes. Gracias a él pude participar en discusiones con Albert Einstein. También estaban presentes Kamerlingh Onnes, Lorentz y Zeeman. Ser un estudiante en manos de tales maestros, es ser en verdad muy afortunado. (citado en O'Connor, et al., 1997)

Tinbergen nació en 1903 y creció en un ambiente que le permitió cultivar un gran sentido de responsabilidad social. Probablemente esto explica su cambio de intereses de la física a la economía durante sus estudios de física teórica en Leiden, que en ese entonces era un pueblo industrial en el que había mucha pobreza, desempleo y serios problemas sociales. Pero sin duda influyó mucho su maestro Ehrenfest, que en años previos había tenido gran interés en la economía, particularmente en las analogías que pudieran utilizarse en el estudio de estas dos disciplinas (Klein, 1985, pp. 305-306), ${ }^{10}$ y quien al ver los intereses de este brillante estudiante no dudó en estimularlo para que profundizara en esos temas y lo asesoró en una tesis que tenía el objetivo de aplicar las metodologías de la física teórica a la teoría económica y que concluyó en 1929. Este original trabajo le permitió marcar un punto

\footnotetext{
${ }^{10}$ Según Klein, en los libros de notas de Ehrenfest, entre octubre de 1917 y mayo de 1918, Ehrenfest había mostrado un gran interés por la posibilidad de desarrollar una analogía entre la termodinámica y la economía, con el concepto de equilibrio como idea central, con la esperanza de que el formalismo de la termodinámica pudiera usarse para lograr nuevas perspectivas en la ciencia económica.
} 
de inflexión en la ciencia económica, introduciendo primero análisis de regresión y correlación, representando la economía como un sistema de ecuaciones diferenciales y examinando posteriormente empíricamente los elementos de causalidad en su teoría. Este trabajo académico de Tinbergen enriqueció sustancialmente la economía como ciencia y le otorgó un mayor prestigio y utilidad (Jolink, 2003).

Finalmente, uno de los últimos estudiantes doctorales de Ehrenfest que gozó de gran éxito profesional, convirtiéndose en uno de los grandes físicos teóricos holandeses, fue Hendrik Casimir. ${ }^{11}$ Ehrenfest tenía a Casimir en gran estima, en el sentido de su capacidad para la física teórica, pues lo consideraba muy intuitivo y poseedor de gran devoción a la física (Lunteren, 2003, p. 37). A su vez, Casimir retrata a Ehrenfest en su pasión y compromiso por la física, refiriéndose a su trabajo y a su obra de la siguiente manera: "Cada una de sus páginas es testigo de su preocupación por la ciencia física. Cada uno de sus trabajos trata de aspectos fundamentales (...) Tiene gran preferencia por el uso de modelos simples que muestren los rasgos esenciales de un problema - y es un maestro inventándolos" (Ehrenfest, 1959, p. XII).

Ehrenfest fue conocido entre la comunidad de físicos teóricos como el 'Sócrates de la Física Moderna'. Es difícil resumir todo lo que Ehrenfest significaba para sus alumnos, pero la siguiente nota de Uhlenbeck cuando, ya trabajando en Ann Arbor, Michigan, en 1930, lo exhorta a aceptar su invitación a un Simposio de Verano de Física Teórica, es muy representativa del aprecio de sus alumnos

¡Se te necesita aquí! (...) Te alegrarás mucho cuando te des cuenta de la manera en que hemos intentado introducir tus ideas aquí, y particularmente de que hemos tenido al menos un poco de éxito. Y para la continuación de nuestro trabajo es realmente muy importante que vengas aquí y que muestres cómo se puede hacer física de una manera amigable y entusiasta, y especialmente cómo un coloquio puede ser inspirador, instructivo y deleitable. No vienes como Profesor Visitante. Si ese fuera el caso, sería fácil encontrar un sustituto. Lo que queremos fundamentalmente, no son tus conferencias, tal vez ni siquiera tu física, sino sobre todo tu atmósfera, tu inspiración, de hecho tu sola presencia. Tu apodo, 'Sócrates de la Física Moderna', es

\footnotetext{
${ }^{11}$ Mejor conocido por el efecto que lleva su nombre (efecto Casimir), que consiste en una débil fuerza que tiende a juntar dos láminas de metal paralelas muy próximas y que tiene una explicación de carácter mecano-cuántica.
} 
espléndido y totalmente exacto. Pero ten en mente que para que tengas gran influencia como Sócrates, debes ser también un filósofo peripatético (citado en Klein, 1989, pp. 41-42). ${ }^{12}$

\section{Ehrenfest: la física y otros intereses}

Comprender verdaderamente la física avanzada era el centro y la razón de ser en la vida de Ehrenfest. Por el tiempo en que él realizaba sus estudios de física, esta disciplina era considerada por algunos como una empresa casi terminada y perfecta. Con la electrodinámica de Maxwell y la descripción mecánica de los fenómenos, se podía explicar casi todo. Pero para una mente inquisitiva como la de Ehrenfest, en la física aún había muchas preguntas por responder. A lo largo de su vida como físico teórico, se interesó por el estado y el papel que representaba el éter como hipótesis de la física que había prevalecido por décadas, por la mecánica estadística como vínculo entre los fenómenos microscópicos y macroscópicos, por las nuevas ideas cuánticas y sus fundamentos y principios, etc.

El momento en que Ehrenfest tomó posesión de su cargo en Leiden se sitúa justamente en el centro de un periodo turbulento para la física. Por un lado, la comunidad de los científicos que trabajaban esta disciplina apenas empezaba a tomar conciencia del significado de la teoría de la relatividad especial de Einstein y, por otro lado, el congreso Solvay de 1911 había traído a la superficie temas que ponían en riesgo las bases mismas de la física. En 1900, el físico alemán Max Planck había presentado una ley que describía la radiación del cuerpo negro, la cual rápidamente fue avalada por datos experimentales. Sin embargo, poco después de 1905, resultó evidente que la derivación de la ley de Planck se basaba en suposiciones extrañas. La interacción entre materia y radiación se desviaba de la noción clásica que explicaba que la fuente de radiación debía poder entregar y recibir energía en forma continua. Con Planck, la energía de la fuente de radiación resultó cuantizada; la energía podía adoptar solo valores fijos, múltiplos de hv (donde $\mathrm{h}$ es la constante de Planck y $v$ la frecuencia de la radiación). Esta cuantización se consideró por un tiempo no como fundamental (incluso por el mismo Planck), sino más bien como artificio matemático para poder concordar con las observaciones. Ehrenfest analizó el trabajo de

\footnotetext{
${ }^{12}$ Carta de Uhlenbeck a Ehrenfest, 5 de mayo de 1930.
} 
Planck en tres publicaciones en 1905, 1906 y $1911 .{ }^{13}$ Este último artículo, que apareció un mes antes del primer congreso Solvay, fue también del conocimiento de Lorentz poco antes de que pusiera su mira en Ehrenfest como su posible sucesor. En ese artículo Ehrenfest mostró la cuantización de la energía como condición necesaria para la derivación de la ley de Planck y, por lo tanto, como propiedad fundamental de la realidad física. Dicho artículo fue además un primer comienzo de lo que se convertiría en la principal aportación de Ehrenfest a la física, es decir, el 'principio adiabático', que junto con el principio de correspondencia de Bohr, buscaría una conexión entre las reglas cuánticas y las teorías tradicionales.

La hipótesis cuántica fue utilizada por el físico danés Niels Bohr para desarrollar una teoría relativa a la estructura atómica. El átomo de Bohr consistía en un núcleo cargado positivamente y electrones cargados negativamente girando alrededor, como ya lo había hecho Rutherford, pero Bohr aplicó adicionalmente reglas cuánticas para la asignación de energía de los electrones. Lo más problemático en la hipótesis de Bohr era su sugerencia de que la frecuencia de la radiación emitida por un átomo no tenía nada que ver con la frecuencia del movimiento periódico de los electrones. Esta suposición iba en contra de la electrodinámica tradicional y de todas las ideas conocidas sobre ondas y vibraciones. De acuerdo con Bohr, la emisión de la radiación era consecuencia de los saltos bruscos de los electrones de una órbita a otra de acuerdo con las reglas cuánticas y la frecuencia de la radiación estaba determinada solamente por las diferencias de energía. Sin embargo, esta suposición absurda le permitía calcular las frecuencias específicas de la radiación espectral de los átomos de hidrógeno. Algunos físicos, entre ellos Einstein y Sommerfeld, tomaron en serio la teoría, impresionados por los resultados que ofrecía. Otros vieron en su derivación solo ideas sin sentido. Ehrenfest se encontraba entre los escépticos. En una carta a Lorentz, confesaba que el trabajo de Bohr lo había llevado a la desesperación: "si ésta es la forma de alcanzar la meta, debo renunciar a hacer física" (citada en Klein, 1985, p. 278). ${ }^{14}$ Por eso, en tanto que Bohr insistía en mostrar la importancia del análisis espectral con el fin de extender la teoría cuántica, Ehrenfest seguía de momento por su propio camino y llegó a escribir en

\footnotetext{
${ }^{13}$ Por las mismas fechas Ehrenfest estuvo muy interesado por los temas de la mecánica estadística, lo cual no es casualidad, pues su interés por indagar los fundamentos del trabajo de Planck seguramente tenían que ver con el uso que éste hacía de la interpretación estadística introducida por Boltzmann y que Ehrenfest conocía muy bien.

${ }^{14}$ Ehrenfest a Lorentz, 25 de agosto de 1913.
} 
1916 una amplia presentación de su principio. Dicho artículo se convirtió en una importante contribución al desarrollo de la teoría cuántica. Sin embargo, después de esa publicación, se apartó del tema por algún tiempo, dejando a su alumno Jan Burgers, que poseía gran habilidad matemática, desarrollara una exploración más profunda de esas ideas (Lunteren, 2003, pp. 16-17).

La pérdida de interés de Ehrenfest por la teoría cuántica se debió probablemente a su desilusión por el hecho de que su trabajo tuvo muy poca resonancia en esos años. Ehrenfest dirigió su interés a otras áreas. Una de ellas fue el problema de la dimensionalidad en física. En 1916, el físico finlandés Gunnar Nordström visitó a Ehrenfest en Leiden. Nordström había publicado recientemente sus ideas en torno a un modelo unificado del universo de cinco dimensiones, y las discusiones que Ehrenfest tuvo con él sobre la generalización de la electrodinámica a un espacio de $\mathrm{N}$ dimensiones lo llevaron a profundizar sobre la cuestión respecto a por qué el espacio es tridimensional (Halpern, 2004, pp. 390400). Dichas investigaciones lo llevaron a publicar un trabajo en 1917 sobre el papel de la tridimensionalidad en la naturaleza, en el que hacía notar que muchos aspectos de las leyes físicas dependían fuertemente del número de dimensiones del espacio (Barrow, 2004, pp. 217-220). Alrededor de la misma época, otro tema que atrajo poderosamente su atención, como ya se mencionó antes en relación con su alumno Tinbergen, fue la teoría económica, intrigado por la posibilidad de desarrollar una analogía entre la termodinámica y la economía. Sin embargo, al término de la guerra ya había vuelto su interés por los temas de la teoría cuántica.

El estímulo para retomar su pasión por los temas de la teoría cuántica fue posiblemente la recepción que Bohr le había dado a su principio adiabático. En una comunicación, Bohr sopesaba el valor del trabajo de Ehrenfest, refiriéndose a "el gran progreso (...) recientemente obtenido por Ehrenfest" (Pais, 1991, p. 190), quien había podido encontrar coherencia a las aparentemente confusas reglas cuánticas. El principio adiabático y su propio principio de correspondencia eran, en opinión de Bohr, las guías para el futuro desarrollo de la teoría cuántica. Tiempo después, en 1925, Ehrenfest dirigió su atención al campo de investigación que antes había sido una de sus pasiones: la mecánica estadística, pero ahora a la luz de la nueva teoría cuántica. En febrero de 1925, Einstein había trabajado en lo que después sería conocido como la 'estadística de Bose-Einstein'. Las partículas perdían su carácter individual, lo que llevaba a un cambio en el enfoque estadístico. 
Ehrenfest se encargó de introducir la interpretación física, en el estilo en que sus colegas estaban acostumbrados (Boeyink, 2005, p. 73).

Si bien la física era su pasión, Paul Ehrenfest también encontró gran placer en el estudio de muy diversos temas. Los intereses de una persona con frecuencia se ven reflejados en lo que expresan desear para sus hijos. Los Ehrenfest amaban a su pequeña hija Tania, tenían un enorme interés en su desarrollo intelectual y se sentían muy orgullosos por su precoz inteligencia. ${ }^{15}$ En cierta ocasión en 1912, Ehrenfest escribió un esquema elaborado de la diversidad de temas que él y su esposa esperaban que la pequeña Tania llegara a conocer (Klein, 1985, pp. 90-91). ${ }^{16}$ La lista era un catálogo de todo lo que en ese momento era excitante en el campo de las ciencias: matemáticas -"todos los aspectos de esta gloriosa ciencia: sus finuras conceptuales, las relaciones entre sus partes más heterogéneas (...) los aspectos puramente metodológicos (...) maestría en las habilidades de cálculo, imaginación geométrica e intuición lógica"-, mecánica, física, química, cristalografía, biología, psicología, economía, etc. Sin embargo, dicho programa educacional no se restringía a las ciencias. Ehrenfest incluyó temas de historia cultural, historia de la ciencia y otras disciplinas. Su interés por temas diversos, a veces alejados de la física, los mostró también en el trato con sus alumnos. En cierta ocasión, después de una conferencia que ofreció en Wageningen en 1919, se embarcó en una discusión con un grupo de estudiantes. Probablemente por su reputación o por algún comentario dentro de la conferencia, salieron a la luz temas de la filosofía de la vida (Lebenphilosophien), que lo llevó a tener con ellos otra reunión más amplia donde discutieron "sobre cuestiones de educación, política y (cosmovisiones) religiosas" (citado en Boeyink, 2005, p. 65). ${ }^{17}$

\section{Ehrenfest: internacionalización y las funciones sociales de la física}

Como veíamos en los apartados anteriores, Ehrenfest realizó aportaciones importantes a la enseñanza de la física en Holanda. Guió a una nueva generación de físicos holandeses y la mayoría de sus estudiantes doctorales se convirtieron en profesores universitarios dentro

\footnotetext{
${ }^{15}$ La primogénita de los Ehrenfest, Tatiana Pavlovna Ehrenfest (su nombre de casada es Tatiana van Aardenne-Ehrenfest), estudió matemáticas y física en la Universidad de Leiden. En 1928 tomó cursos en Gotinga bajo la dirección de Harald Bohr y Max Born. Obtuvo su doctorado en Leiden en 1931. En el campo de las matemáticas se le reconoce por el teorema BEST, (de Brujin, van AardenneEhrenfest, Smith y Tutte).

${ }_{17}^{16}$ Klein toma la información de un libro familiar de los Ehrenfest.

${ }^{17}$ Ehrenfest a Bohr, 4 de junio de 1919.
} 
de las mismas fronteras holandesas o en el extranjero. Ehrenfest se convirtió además en uno de los nodos de la red de físicos europeos preocupados por la nueva teoría cuántica y contribuyó regularmente a las reuniones internacionales que se organizaron sobre la teoría atómica. Sin embargo, su influencia no se limitó a estos aspectos del desarrollo de su disciplina, sino que también se preocupó por el papel de la ciencia en la sociedad y llevó a cabo acciones locales dirigidas hacia una reforma de la universidad (Lunteren, 2004).

En 1891 se había fundado en Eindhoven, al sur de Holanda, la compañía Philips, cuyo giro inicial fue la producción de lámparas incandescentes. En 1910 había establecido su propio laboratorio de investigación (NatLab). Paul Ehrenfest y Leonard S. Ornstein (catedrático en Utrecht) fueron nombrados consultores académicos en el NatLab, con lo cual Ehrenfest pudo jugar un papel activo en la interacción entre la universidad y la industria (Boeyink, 2005, p. 64). Al principio, en los años 20, esta situación representó para Ehrenfest un apoyo económico, pues obtenía un ingreso extra organizando una serie de conferencias en el NatLab. Con el transcurso del tiempo, fue afirmándose en él la convicción de que era importante formar no solo físicos teóricos fielmente consagrados a la ciencia pura, cuya preocupación principal tuviera que ver con principios esotéricos y abstractos en física atómica, sino también profesionales preparados para atender los nuevos laboratorios industriales e interesados en comprender los métodos particulares de investigación usados en ellos. Este cambio de actitud respecto a la relación entre la ciencia, la universidad y la sociedad lo llevó a promover en 1928 la candidatura de Gilles Holst, quien era desde 1916 el director del NatLab, para un puesto especial de profesor.

Sin embargo, ese cambio de visión de Ehrenfest respecto al papel de la universidad en la sociedad, no se debió solamente a su relación con la compañía Philips. Probablemente fue más decisiva su visita a los Estados Unidos en 1923-1924, donde había sido invitado por Robert Millikan a dar unas conferencias en el California Institute of Technology, invitación a la cual Ehrenfest accedió pensando en la posibilidad de poder establecer contactos que resultaran beneficiosos para los nuevos físicos holandeses. ${ }^{18}$ Ehrenfest quedó asombrado por el carácter práctico de las universidades estadounidenses y por los mecanismos de enlace entre la investigación y las necesidades sociales, lo cual contrastaba mucho con el

\footnotetext{
${ }^{18}$ De hecho, algún tiempo después de su visita a los Estados Unidos, su alumno Gerhard Diele se fue a Berkeley a trabajar con el espectroscopista estadounidense Birge y poco después George Uhlenbeck y Samuel Goudsmit obtuvieron cargos como profesores en la Universidad de Michigan.
} 
carácter de las universidades holandesas (o europeas en general). Aunque en un principio Ehrenfest creía que la física debía estudiarse por su valor intrínseco como ciencia pura, después fue apreciando muchos aspectos de la vida académica estadounidense. En su segunda visita a los Estados Unidos, en 1930, quedó aún más impresionado por su sistema y urgió a las autoridades de la Universidad de Leiden a realizar algunas reformas a la misma. Aunque en opinión de algunos, incluyendo el presidente del consejo, las universidades de los Estados Unidos carecían de profundidad cultural, resultaba claro que en el campo de las ciencias sí era necesaria una mayor cooperación con la industria. En el mismo año de 1930 se había fundado el Instituto Nacional para la Ciencia Aplicada, cuya función era actuar como intermediaria entre la universidad y la industria, pero Ehrenfest y Hugo Kruyt (químico de la Universidad de Utrecht) eran de la idea de que esto era solo un primer paso para abandonar el aislamiento de las universidades holandesas y permitir que la industria se beneficiara del conocimiento más fundamental que ofrecía la ciencia.

Además, en términos más generales, Ehrenfest abrigaba la esperanza de que la comunidad internacional de físicos sirviera de ejemplo de hermandad internacional, pues dentro de ella se hablaba un lenguaje común y se seguían reglas de comportamiento racional. En la visión de Ehrenfest, si la economía y la política se desarrollaran con base en principios científicos, podrían ayudar a crear y dar forma a la estructura del estado futuro, es decir, defendía una visión tecnocrática de los futuros gobiernos.

De esta manera, Ehrenfest, sin haber crecido en Holanda, tuvo el privilegio de dejar huella en el desarrollo de la física holandesa y de fomentar su relación con la industria y la sociedad.

\section{Desesperanza, tragedia y muerte de Ehrenfest}

Ehrenfest se vio toda su vida abrumado por sentimientos de incertidumbre respecto a su desempeño como investigador, y en sus últimos años (después de 1926), también respecto a su labor como profesor. No solo se sintió incapaz de ofrecer alguna contribución a la teoría cuántica, sino, además, ni siquiera poder dar seguimiento a su acelerado y caótico desarrollo. Las nuevas teorías tenían un nivel de abstracción mucho mayor que la vieja teoría cuántica. La posibilidad de visualización de los fenómenos había cedido su sitio al uso de complejas operaciones matemáticas. En tanto que muchos físicos pertenecientes al viejo 
orden ignoraron los nuevos desarrollos tanto como les fue posible, a Ehrenfest lo llevaban a la desesperación, haciéndolo sentir que había perdido el rumbo (Lunteren, 2003, p. 31).

Los problemas de Ehrenfest para sostenerse ante la situación que guardaba la física, su disciplina, se agravaron con la muerte de Lorentz en 1928 y por el gradual distanciamiento de su esposa. Ella pasaba largas temporadas en Rusia, pero la separación no era solo geográfica, por eso, tuvieron la intención de disolver su matrimonio, aunque dicha intención nunca se llevó a cabo.

Ehrenfest anhelaba cancelar su trabajo en Leiden e incluso concluir su 'inútil' vida. Lo único que lo sostenía era que sus cuatro hijos dependían de su ingreso. El menor de ellos, Vassily, había sido diagnosticado con síndrome de Down y Ehrenfest deseaba evitar que su cuidado fuera una carga para sus otros tres hijos (Tania, Galya y Paul). (Lunteren, 2003, p. 32). Buscó con su amigo Joffe, quien ahora contaba con una posición prominente en el mundo científico de Rusia, una nueva posición en la Unión Soviética. Su aspiración era construir una nueva vida, pero las cosas no resultaron de acuerdo a los planes. Así, el destino de Ehrenfest parecía estar ya definido. En el verano de 1932 había escrito una carta de despedida a sus mejores amigos (Bohr, Einstein, Herglotz, Joffe y Kohnstamm), la cual nunca fue enviada y en la que explicaba que el suicidio era su única alternativa.

En julio de 1933, aparentemente hubo una mejora en su estado de ánimo. Incluso desarrolló algunos planes con Casimir sobre algunas investigaciones a realizar. En agosto pasó unos días en Schiermonnikoog y en septiembre estuvo unos días en Copenhague. Pero no sirvió de nada. En Copenhague dejó una impresión extraña al concluir su visita. Dirac recordó, posteriormente, haber escuchado una rápida alusión sobre el fin de su vida. Ya de regreso en Holanda, y habiendo perdido la batalla contra la depresión que lo agobiaba, viajó el 25 de septiembre al Wateringinstituut en Ámsterdam, donde estaba siendo tratado su hijo Vassily. Después de convivir con él una hora, tomó un arma, le disparó primero a su hijo y enseguida a él mismo, poniendo fin a un largo calvario.

\section{Conclusiones}

El progreso científico, particularmente dentro de la física, se ha visto de vez en cuando ensombrecido cuando el conocimiento ya no puede avanzar más dentro de los paradigmas de entendimiento asimilados o aceptados en un momento dado de la historia, como ocurrió con la época de crisis, no solo científica sino también en otros aspectos culturales, que 
aconteció en los inicios del siglo XX. En ese contexto, Paul Ehrenfest fue el físico por excelencia que discutió apasionadamente con sus colegas y discípulos los problemas que surgían en su ciencia.

Si bien Hendrik Lorentz y Kamerlingh Onnes le habían otorgado a la Universidad de Leiden fama internacional en el campo de la física, Ehrenfest fue quien aseguró la continuación de dicha reputación científica. Como parte de esta tarea Ehrenfest logró que muchos científicos extranjeros visitaran o tuvieran algún tipo de estancia académica en Leiden y que de sus aulas surgieran físicos brillantes, a quienes impulsó a través de un contacto muy personal, y que tendrían una reconocida carrera científica en diversos campos.

Finalmente, resulta provechoso, como lo hemos mostrado en este trabajo, vincular las diferentes facetas de un hombre de ciencia como Paul Ehrenfest en función de otros elementos socioculturales relacionados con la educación, la industria, el estado. A la luz de los modernos modelos de vinculación academia-industria-estado-sociedad para el desarrollo de los países y de las regiones, resulta interesante e instructivo mirar al pasado, en momentos más preliminares del proceso de institucionalización de la ciencia y encontrar ahí sus formas más incipientes y a sus primeros protagonistas y propulsores.

\section{REFERENCIAS}

Barrow, John. (2004). The Constants of Nature: The Numbers that Encode the Deepest Secrets of the Universe. New York: Vintage Books.

Boeyink, Rowdy. (2005). In het centrum van het drama: Wetenschappelijke worstelingen van Paul Ehrenfest tussen 1916 - 1925. (In the center of the drama: Scientific struggles of Paul Ehrenfest between 1916 - 1925). Doctoraalscriptie, Universiteit Utrecht.

Cohen, Ezechiel Godert David. (1990). George E. Uhlenbeck and statistical mechanics, American Journal of Physics, 58(7), 619-625.

Goudsmit, Samuel A. (1971). De ontdekking van de electronenrotatie. Nederlands Tijdschrift voor Natuurkunde 37, 386. Recuperado de: http://www.lorentz.leidenuniv.nl/history/spin/goudsmit.html

Halpern, Paul. (2004). Nordström, Ehrenfest, and the Role of Dimensionality in Physics. Physics in Perspectiva, 6(4), 390-400.

Jolink, Albert J. (2003). Jan Tinbergen. The Statistical Turn in Economics: 1903-1955. Rotterdam: Chimes. 
Jungnickel, Christa y McCormmach, Russell. (1990). Intellectual Mastery of Nature, Vol. II: The Now Mighty Theoretical Physics 1870-1925. Chicago: The University of Chicago Press.

Klein, Martin J. (ed). (1959). Paul Ehrenfest Collected Scientific Papers. Amsterdam: North-Holland Publishing Company.

Klein, Martin J. (1981). Not by discoveries alone: The centennial of Paul Ehrenfest. Physica A (Elsevier), 106, 3-14.

Klein, Martin J. (1985). Paul Ehrenfest: The making of a theoretical physicist. Amsterdam: North-Holland Physics Publishing.

Klein, Martin J. (1989). Physics in the Making in Leiden: Paul Ehrenfest as Teacher. En Sarlemijn, A. y Sparnaay, M.J. (Eds.), Physics in the Making: Essays on Developments in 20th Century Physics (pp. 29-44). Amsterdam: North-Holland Publishing Company.

Lunteren, Frans van. (2003). Paul Ehrenfest: de Leidse onderzoekschool van een fysicus in diaspora. (Paul Ehrenfest: the Leiden research school of a physicist in diaspora). Recuperado de: http://www.rowdyboeyink.org/ehrenfest/images/Ehrenfest2.doc

Lunteren, Frans van. (2004). Paul Ehrenfest and Dutch physics in the interwar period. Recuperado de: http://www.rowdyboeyink.org/ehrenfest/images/Vancouver2.doc

O'Connor, John J. y Robertson, Edmund F. (1997). Jan Tinbergen. Recuperado de: http://www-groups.dcs.st-and.ac.uk/ history/Printonly/Tinbergen.html

Pais, Abraham. (1991). Niels Bohr's Times, in physics, philosophy, and polity. New York: Oxford University Press.

The Burgers Program for Fluid Dynamics. Recuperado de: http://www.burgers.umd.edu/about.html

Uhlenbeck, George E. (1956). Reminiscences of Professor Paul Ehrenfest. American Journal of Physics, 24(6), 431-433. 